\title{
Observer-reported Outcome
}

National Cancer Institute

\section{Source}

National Cancer Institute. Observer-reported Outcome. NCI Thesaurus. Code C142620.

A clinical outcome assessment that is reported by someone other than the patient or a healthcare professional. 